\title{
A rare case of paradoxical embolism
}

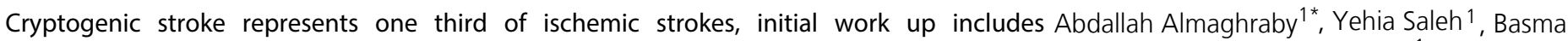
Computed Tomography (CT), Magnetic resonance imaging (MRI), laboratory investigations, Hammad, Mahmoud Abdelnaby, Ashraf ElAmin Electrocardiogram (ECG) and Echocardiography either transthoracic (TTE) or Transesophageal Mohamed Kandil ${ }^{1}$, Haitham Badran ${ }^{3}$ and (TEE). Patent foramen ovale (PFO) which is seen in between $15 \%$ and $25 \%$ of adults has been Mohamed Abdel-Hay ${ }^{1}$ identified as a source for cryptogenic ischemic stroke.

${ }^{1}$ Department of Cardiology, University of Alexandria, Egypt

${ }^{2}$ Massachusetts General Hospital, Boston, USA

Keywords: Embolism; Cryptogenic stroke

${ }^{3}$ Department of Cardiology, University of Ain Shams, Egypt

${ }^{*}$ Author for correspondence:

dr.maghraby@gmail.com

Tel: +2035921675

\section{Introduction}

Stroke is the number one cause of disability in developed countries and the third cause of mortality and up to $15-30 \%$ of ischemic strokes are caused by a cardiac source of emboli being associated with poor prognosis and a high rate of mortality [1].

Cryptogenic stroke represents one third of ischemic strokes, initial work up includes Computed Tomography (CT), Magnetic resonance imaging (MRI), laboratory investigations, Electrocardiogram (ECG) and Echocardiography either transthoracic (TTE) or Transesophageal (TEE) [2].

Patent foramen ovale (PFO) which is seen in between $15 \%$ and $25 \%$ of adults has been identified as a source for cryptogenic ischemic stroke [3].

The prevalence of PFO has been shown to be higher in young adults with cryptogenic stroke. In this population, PFO and deep vein thrombosis (DVT) are both common concomitant findings [4].

\section{Case Report}

A 61-year-old male patient with no previous medical history presented to our hospital complaining of rapidly progressive dyspnoea over 2 days, no orthopnoea, no paroxysmal nocturnal dyspnoea, no chest pain.

His clinical examination showed tachycardia 110 beats per minute, his blood pressure was $90 / 60 \mathrm{mmHg}$, his respiratory rate was 24 cycles per minute and his Oxygen caturation was $90 \%$ on facemask. Heart and Submitted: 19 January 2017 chest examinations were unremarkable and Published online: 10 February 2017 tender right calf muscles was evident on lower limb examination.

Laboratory work-up showed elevated Ddimer and leucocytosis, urgent duplex of both lower limbs showed extensive fresh deep venous thrombosis (DVT), urgent multi-slice computed tomography (MSCT) of the pulmonary arteries showed extensive bilateral pulmonary thrombo-embolization of both right and left main branches.

On admission to the cardiac care unit (CCU), the patient started to be more hemodynamically unstable and his blood pressure fell down to $70 / 40 \mathrm{mmHg}$, so a decision of thrombolytic administration was taken immediately (Figures 1-3).

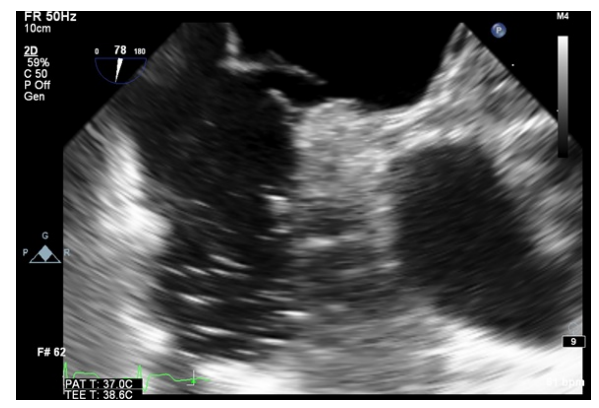

Figure 1: 2D TEE showing the PFO just before the injection of agitated saline. 


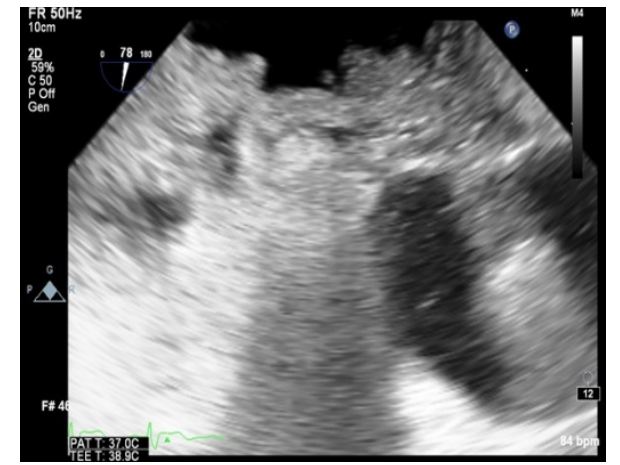

Figure 2: 2D TEE with agitated saline showing its passage from the right atrium to the left atrium through the PFO.

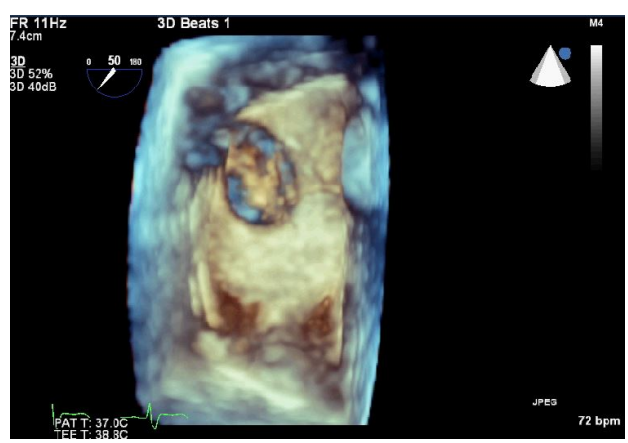

Figure 3: 3D TEE from the right atrium prospective showing the PFO with its ovoid shape and incomplete margins.

Suddenly before thrombolytic administration the patient developed dysarthria with evident lag on his right side, also on rapid examination his right and left radial pulses became absent but his lower limb pulses were well felt.

Urgent computed tomography angiography (CTA) for both upper limbs and the brain was done immediately and revealed right upper limb total thrombotic occlusion at the level of brachial artery, left upper limb total occlusion at the level of the proximal radial artery and unremarkable brain imaging.
Urgent neurological and vascular surgery consultations were sought immediately and they gave a decision of a conservative strategy without an objection to thrombolytic administration. Alteplase was given through a right internal jugular central line with great improvement of clinical signs.

Transthoracic echocardiography revealed good left ventricular systolic function, right ventricular strain with signs of acute pulmonary embolism, and evidence of small inter-atrial septal defect with intermittent color flow in both directions.

Intravenous agitated saline was injected through the central venous line several times till evidence of right to left shunt through an atrial septal defect or patent foramen ovale (PFO) was detected.

After complete stabilization of his hemodynamics one day later, a transesophageal echocardiography revealed a large PFO with intermittent right to left shunt on intravenous agitated saline injection. Few days later the patient was scheduled for PFO closure via Amplatzer occluder that was done successfully at another center.

\section{Discussion}

To our knowledge, this is the first reported case of simultaneous paradoxical thrombo-embolization in three different systemic sites.

Usually the most common site for paradoxical embolization is the cerebral circulation causing cryptogenic stroke [5].

\section{Conclusion}

Diagnosis of paradoxical stroke is a clinical diagnosis and imaging modalities are only used as a confirmatory tool and guidance for therapy.

\section{Executive Summary}

Cryptogenic stroke represents one third of ischemic strokes, initial work up includes Computed Tomography (CT), Magnetic resonance imaging (MRI), laboratory investigations, Electrocardiogram (ECG) and Echocardiography either transthoracic (TTE) or Transesophageal (TEE).

Patent foramen ovale (PFO) which is seen in between $15 \%$ and $25 \%$ of adults has been identified as a source for cryptogenic ischemic stroke.

\section{References}

1. Ustrell X, Pellise A. Cardiac Workup of Ischemic Stroke. Cur. Cardiol. Rev. 6(3), 175-183 (2010).

2. Yaghi S, Elkind MSV. Cryptogenic stroke: A diagnostic challenge. Neurology: Clin. Prac. 4(5), 386-393 (2014).
3. Lechat P, Mas JL, Lascault G, et al. Prevalence of patent foramen ovale in patients with stroke. N. Engl. J. Med. 318 $1148-1152$ (1988)

4. Smajlović D. Strokes in young adults: epidemiology and prevention. Vascul. Heal. Risk. Manag. 11, 157-164 (2015).

5. Windecker S, Stortecky S, Meier B. Paradoxical Embolism. J. Am. Coll. Cardiol. 64(4), 403-415 (2014). 